Ferrata Storti Foundation

\title{
Clinical significance of soluble CADM1 as a novel marker for adult T-cell leukemia/lymphoma
}

\author{
Shingo Nakahata, ${ }^{1 *}$ Syahrul Chilmi, ${ }^{1 *}$ Ayako Nakatake, ${ }^{1}$ Kuniyo Sakamoto, ${ }^{1}$ \\ Maki Yoshihama, ${ }^{1}$ Ichiro Nishikata, ${ }^{1}$ Yoshinori Ukai, ${ }^{2}$ Tadashi Matsuura, ${ }^{2}$ \\ Takuro Kameda, ${ }^{3}$ Kotaro Shide, ${ }^{3}$ Yoko Kubuki, ${ }^{3}$ Tomonori Hidaka, ${ }^{3}$ \\ Akira Kitanaka, ${ }^{4}$ Akihiko Ito, ${ }^{5}$ Shigeki Takemoto, ${ }^{6}$ Nobuaki Nakano, ${ }^{7}$ \\ Masumichi Saito, ${ }^{8}$ Masako Iwanaga, ${ }^{9}$ Yasuko Sagara, ${ }^{10}$ Kosuke Mochida, ${ }^{11}$ \\ Masahiro Amano, ${ }^{11}$ Kouichi Maeda, ${ }^{12}$ Eisaburo Sueoka, ${ }^{13}$ Akihiko Okayama, ${ }^{14}$ \\ Atae Utsunomiya, ${ }^{7}$ Kazuya Shimoda, ${ }^{3}$ Toshiki Watanabe ${ }^{15}$ \\ and Kazuhiro Morishita ${ }^{1}$
}

Volume 106(2):532-542

\begin{abstract}
${ }^{1}$ Division of Tumor and Cellular Biochemistry, Department of Medical Sciences, Faculty of Medicine, University of Miyazaki, Miyazaki; ${ }^{2}$ Perseus Proteomics Inc., Perseus Proteomics Inc., Tokyo; ${ }^{3}$ Division of Gastroenterology and Hematology, Department of Internal Medicine, Faculty of Medicine, University of Miyazaki, Miyazaki; ${ }^{4}$ Department of Laboratory Medicine, Kawasaki Medical School, Okayama; ${ }^{5}$ Department of Pathology, Kindai University School of Medicine, Osaka; ${ }^{6}$ National Hospital Organization Kumamoto Medical Center, Kumamoto; 'Department of Hematology, Imamura General Hospital, Kagoshima, ${ }^{8}$ Department of Safety Research on Blood and Biological Products, National Institute of Infectious Diseases, Tokyo; ${ }^{9}$ Department of Frontier Life Science, Nagasaki University Graduate School of Biomedical Sciences, Nagasaki; ${ }^{10}$ Japanese Red Cross Kyushu Block Blood Center, Fukuoka; ${ }^{11}$ Department of Dermatology, Faculty of Medicine, University of Miyazaki, Miyazaki; ${ }^{12}$ Internal Medicine, National Hospital Organization Miyakonojo Medical Center, Miyazaki; ${ }^{13}$ Department of Laboratory Medicine, Saga University Hospital and Department of Clinical Laboratory Medicine, Faculty of Medicine, Saga University, Saga; ${ }^{14}$ Department of Rheumatology, Infectious Diseases and Laboratory Medicine, University of Miyazaki, Miyazaki and ${ }^{15}$ Department of Computational Biology and Medical Sciences, Graduate School of Frontier Sciences, The University of Tokyo, Tokyo, Japan

*SN and CS contributed equally as co-first authors.

${ }^{\circ}$ Current address: JURAKU Internal Medicine Clinic, Kumamoto, Japan.
\end{abstract}

\section{ABSTRACT}

\section{Correspondence:}

KAZUHIRO MORISHITA

kmorishi@med.miyazaki-u.ac.jp

Received: August 21, 2019.

Accepted: February 7, 2020.

Pre-published: February 13, 2020.

https://doi.org/10.3324/haematol.2019.234096

(C)2021 Ferrata Storti Foundation

Material published in Haematologica is covered by copyright. All rights are reserved to the Ferrata Storti Foundation. Use of published material is allowed under the following terms and conditions:

https://creativecommons.org/licenses/by-nc/4.0/legalcode. Copies of published material are allowed for personal or internal use. Sharing published material for non-commercial purposes is subject to the following conditions:

https://creativecommons.org/licenses/by-nc/4.0/legalcode, sect. 3. Reproducing and sharing published material for commercial purposes is not allowed without permission in writing from the publisher.
$\Delta$ dult T-cell leukemia/leukemia (ATLL) is an aggressive peripheral T-cell malignancy, caused by infection with the human T-cell leukemia virus type 1 (HTLV-1). We recently showed that the cell adhesion molecule 1 (CADM1), a member of the immunoglobulin superfamily, is specifically and consistently overexpressed in ATLL cells, and functions as a novel cell surface marker. In this study, we first show that a soluble form of CADM1 (sCADM1) is secreted from ATLL cells by mainly alternative splicing. After developing the Alpha linked immunosorbent assay (AlphaLISA) for sCADM1, we show that plasma sCADM1 concentrations gradually increased during disease progression from indolent to aggressive ATLL. Although other known biomarkers of tumor burden such as soluble interleukin-2 receptor $\alpha$ (sIL-2R $\alpha$ ) also increased with sCADM1 during ATLL progression, multivariate statistical analysis of biomarkers revealed that only plasma sCADM1 was selected as a specific biomarker for aggressive ATLL, suggesting that plasma SCADM1 may be a potential risk factor for aggressive ATLL. In addition, plasma SCADM1 is a useful marker for monitoring response to chemotherapy as well as for predicting relapse of ATLL. Furthermore, the change in SCADM1 concentration between indolent and aggressive type ATLL was more prominent than the change in the percentage of $\mathrm{CD} 4^{+} \mathrm{CADM1} 1^{+} \mathrm{ATLL}$ cells. As plasma sCADM1 values fell within normal ranges in HTLV-1-associated myelopathy/tropical spastic paraparesis (HAM/TSP) patients with higher levels of serum sIL-2R $\alpha$, the measurement of sCADM1 may become a useful tool to discriminate between ATLL and other inflammatory diseases, including HAM/TSP. 


\section{Introduction}

Adult T-cell leukemia/lymphoma (ATLL) is a refractory $\mathrm{CD}^{+} \mathrm{T}$-cell malignancy associated with human T-cell leukemia virus type 1 (HTLV-1) ${ }^{1.3}$ It is estimated that $15-$ 20 million people are currently infected with HTLV-1 worldwide, and a high prevalence of HTLV-1 infection can be found in many parts of the world, including southwestern Japan, Melanesia, South America, sub-Saharan Africa, the Caribbean, Romania, central parts of Australia, and the Middle East. ATLL is classified into four subtypes acute, lymphoma, chronic, and smoldering type. Patients with indolent ATLL (chronic or smoldering) have a better prognosis and watchful waiting or combined zidovudine (AZT) and interferon- $\alpha$ (IFN- $\alpha$ ) therapy is standard treatment for indolent disease. Patients with aggressive forms (acute and lymphoma) have a very poor prognosis due to the intrinsic chemotherapy resistance of malignant cells. Allogeneic hematopoietic stem-cell transplantation (allo-HSCT), mogamulizumab, an anti-CC chemokine receptor 4 (CCR4) monoclonal antibody, or AZT/IFN therapy are important for the treatment of ATLL; however, the prognosis is unfavorable in many cases. ${ }^{47}$

Serum levels of interleukin-2 receptor $\alpha$ (sIL-2R $\alpha$, sCD25) are known to reflect tumor burden due to the high expression levels of IL-2R on ATLL cells. ${ }^{8}$ However, SIL2R levels are also increased during an inflammatory response related to HTLV-1-associated myelopathy/tropical spastic paraparesis (HAM/TSP), ${ }^{9}$ or graft-versus-host disease $(\mathrm{GvHD})^{10,11}$ that is often seen after mogamulizum$\mathrm{ab}$ and allo-HSCT treatment; ${ }^{12-14}$ consequently, ATLL reoccurrence and inflammatory responses may not be distinguishable. The elevation of sIL2R levels has also been reported in various hematologic and solid malignancies. ${ }^{15,16}$ Therefore, the development of a specific and reliable diagnostic marker for ATLL is of great clinical significance.

CADM1 was originally isolated as a tumor suppressor gene in non-small cell lung cancer, and functions in the cell-cell adhesion of endothelial cells. ${ }^{17,18}$ We found that CADM1 was ectopically and highly expressed in HTLV-1infected T cells and ATLL cells, resulting in the enhanced adhesion of ATLL cells to promote the invasion of ATLL cells into various organs..$^{19,20}$ As CADM1 is consistently and specifically expressed in HTLV-1-infected T cells and ATLL cells, ${ }^{21-23}$ but not in most of the non-ATLL lymphomas, ${ }^{21,2426}$ CADM1 is now thought to be the best cell surface marker for ATLL.

sCADM1 consists of the extracellular domain of CADM1, which is generated by alternative splicing of CADM1 pre-mRNA ${ }^{27,28}$ or shedding of CADM1 protein on the cell surface. ${ }^{29-31}$ We have previously shown that sCADM1 protein is detected in the serum of acute-type ATLL patients. ${ }^{21}$ In the present study, we developed a highly sensitive and efficient method for the measurement of sCADM1 using the Alpha linked immunosorbent assay (AlphaLISA) technology. ${ }^{32,33}$ sCADM1 levels were found to be increased in smoldering to acute type ATLL, which is highly correlated with various clinical parameters, including serum sIL-2 $\alpha$ levels. Furthermore, sCADM1 levels correlated with the leukemic cell burden in ATLL patients during the course of chemotherapy treatments. These results suggest that $\mathrm{SCADM1}$ can be a specific biomarker for ATLL, and that a measurement of sCADM1 may become a useful tool for accurately predicting leukemic cell burden and the disease progression or status of ATLL patients.

\section{Methods}

\section{Patient samples}

Peripheral blood samples were collected from HTLV-1 carriers $(n=94)$, patients with smoldering-type $(n=80)$, chronic-type $(n=70)$, acute-type $(n=71)$, and lymphoma-type ( $n=37)$ ATLL, patients with HAM/TSP $(\mathrm{n}=12)$, and healthy volunteers as controls ( $\mathrm{n}=35$ ) (Online Supplementary Table S1). These samples were obtained from Miyazaki University Hospital (Miyazaki, Japan), National Hospital Organization Miyakonojo Medical Center (Miyazaki, Japan), Imamura General Hospital (Kagoshima, Japan), National Hospital Organization Kumamoto Medical Center (Kumamoto, Japan), and the Group of Joint Study on Predisposing Factors of ATL Development (JSPFAD, Japan). Informed consent was obtained from all patients. This study was approved by the Institutional Review Board at the Faculty of Medicine, University of Miyazaki, in accordance with the Declaration of Helsinki. Diagnosis of ATLL was based on clinical features, hematological characteristics, serum antibodies against HTLV-1, and monoclonal integration of the HTLV-1 proviral genome. Plasma and serum samples were prepared by centrifugation and stored at $-80^{\circ} \mathrm{C}$ until use. Peripheral blood mononuclear cells (PBMC) were isolated by Histopaque density gradient centrifugation (Sigma-Aldrich, Tokyo, Japan) according to the manufacturer's protocol. The procedures for purification of ATLL cells from patients by using antiCADM1-antibody-coated magnetic beads have been described elsewhere. ${ }^{21} \mathrm{CD}^{+} \mathrm{T}$ cells from healthy volunteers were purified from PBMC using microbeads (Miltenyi Biotec, Auburn, CA, USA). Purity of isolated $\mathrm{CD}^{+} \mathrm{CADM}^{+}$cell populations from ATLL patients was confirmed by flow cytometry. ${ }^{21}$

\section{Cell lines}

The HTLV-1-negative human T-cell acute lymphoblastic leukemia (T-ALL) cell line MOLT4, the cutaneous T-cell lymphoma (CTCL) cell line HUT78, the HTLV-1-infected T-cell line MT2, and ATLL-derived cell lines (S1T and ST1) were maintained in RPMI 1640 medium (Wako, Osaka, Japan) supplemented with $10 \%$ fetal bovine serum (FBS) and $50 \mu \mathrm{g} / \mathrm{mL}$ of penicillin/streptomycin in a $5 \% \mathrm{CO}_{2}$ chamber at $37^{\circ} \mathrm{C}$. The IL-2-dependent ATLLderived cell lines $\mathrm{KK} 1$ and $\mathrm{KOB}$ were maintained in complete RPMI 1640 medium supplemented with $0.75 \mathrm{~g} / \mathrm{mL}$ of recombinant human IL-2 (Peprotech, Rocky Hill, NJ, USA). The human osteosarcoma cell line Saos-2 was cultured in Dulbecco's modified Eagle's medium (DMEM, Wako) supplemented with 10\% FBS and $50 \mu \mathrm{g} / \mathrm{mL}$ of penicillin/streptomycin. MOLT4 was obtained from the Fujisaki Cell Center, Hayashibara Biochemical Laboratories (Okayama, Japan). MT2 was kindly provided by Dr. H. Iha (Oita University, Japan). ST1, KOB, and KK1 were kindly provided by Dr. Y. Yamada (Nagasaki University, Japan). S1T was a kind gift from Dr. N. Arima (Kagoshima University, Japan). Saos-2 was obtained from the RIKEN BioResource Center (Tsukuba, Japan).

\section{Measurement of SCADM1 concentrations in the blood by AlphaLISA}

The anti-CADM1 antibody (103-109) was generated by phagedisplay technology. ${ }^{34}$ AlphaLISA was performed in 96-well microtiter plates containing $5 \mu \mathrm{L}$ of plasma sample, $10 \mu \mathrm{L}$ of biotinylated anti-CADM1 antibody (3E1, MBL, Nagoya, Japan) $(0.1 \mathrm{nM}),{ }^{10} \mathrm{~L}$ of $10 \mu \mathrm{g} / \mathrm{mL}$ anti-CADM1 antibody (103-109) -conjugated AlphaLISA acceptor beads $(10 \mu \mathrm{g} / \mathrm{mL})$, and $25 \mu \mathrm{L}$ of streptavidin-coated AlphaLISA donor beads $(40 \mu \mathrm{g} / \mathrm{mL})$ in AlphaLISA 
ImmunoAssay Buffer (25 mM HEPES, pH 7.4, 0.1\% Casein, 0.5\% Triton X-100, $1 \mathrm{mg} / \mathrm{mL}$ Dextran-500, and $0.05 \%$ Proclin-300). The reaction mixture was incubated at $23^{\circ} \mathrm{C}$ for 1 hour (h) and the chemical emission was read on an EnSpire Alpha microplate reader (PerkinElmer Waltham, MA). The concentrations of sCADM1 were obtained from a standard curve generated by recombinant CADM1 protein (Sino Biological, Beijing, China) using four-parameter logistic curve fitting, and multiplied by the dilution factor. All measurements were done in duplicate.

Additional methods are provided in the Online Supplementary Appendix.

\section{Results}

\section{The soluble form of CADM1 mRNA is efficiently expressed in ATLL}

As sCADM1 can be generated through alternative splicing by an intron retention event ${ }^{27,28}$ or ectodomain shedding of CADM1 protein, ${ }^{29.31}$ the mRNA expression of a soluble splice variant of CADM1 and the level of CADM1 shedding were initially determined in ATLL-related cell lines and leukemia cells from patients with acute-type ATLL by RT-PCR and western blotting, respectively. Because the sCADM1 transcript contains the 118-bp sequence of intron 7 (Figure 1A), ${ }^{27}$ we used PCR primers located in exon 6 and intron 7 of CADM1 to amplify the sCADM1 transcript. Semiquantitative RT-PCR showed that $s C A D M 1$ mRNA, along with the membrane-bound form of CADM1 (mbCADM1) mRNA, was highly expressed in all of the HTLV-1-positive ATLL-related cell lines (Online Supplementary Figure S1A). We then performed quantitative RT-PCR analysis with purified $\mathrm{CD} 4^{+} \mathrm{CADM1}{ }^{+}$leukemic cell populations from various types of ATLL patients. ${ }^{21}$ A significantly higher abundance of $s C A D M 1$ mRNA was observed in all ATLL samples tested, including smoldering, chronic, and acute-type ATLL, compared with $\mathrm{CD}^{+} \mathrm{T}$ cells from healthy volunteers (Figure 1B). Similarly, the levels of $m b C A D M 1$ or total CADM1 (tCADM1) were significantly higher in all subtypes of ATLL than controls (Figure 1B). In order to quantify CADM1 shedding in ATLL, we analyzed the levels of two membrane-associated C-terminal fragments $(\alpha C T F, 18 \mathrm{kDa}$ and CTF, $35 \mathrm{kDa}$ ), which are generated by the proteolytic cleavage of CADM1 by ADAM and unidentified proteases, respectively. ${ }^{29-31}$ Although the CTF fragment of CADM1 was detected in ATLL-related cell lines, very weak or no signal was found in leukemia cell samples from ATLL patients (Online Supplementary Figure $S 2 A-B)$. Additionally, CADM1 undergoes alternative splicing between exons 7 and 11 (Figure 1A) and the inclusion of exon 9 confers shedding susceptibility to CADM $1 .{ }^{31}$ RTPCR analysis revealed that the majority of the $m b C A D M 1$ mRNA skipped both exons 9 and 10 (Online Supplementary Figure $S 1 A-B)$. Therefore, these results suggest that upregulated expression of $s C A D M 1 \mathrm{mRNA}$, which is generated by retention of intron 7 , might be the main cause of the increased level of sCADM1 protein in ATLL.

\section{Plasma SCADM1 levels increase with disease progression in ATLL}

In order to investigate the clinical utility of sCADM1 in the diagnosis of ATLL patients, we developed a highly sensitive method for the measurement of plasma sCADM1 with the use of the AlphaLISA technology, which is based on energy transfer from a streptavidin donor bead to an AlphaLISA acceptor bead in close proximity. 22,33 The donor and the acceptor beads were conjugated with biotinylated anti-CADM1 (3E1) and anti-CADM1 (103-189) antibodies, respectively (see Methods). Using recombinant human CADM1 protein, the assay system was capable of detecting sCADM1 in the plasma at concentrations as low as $\sim 0.2 \mathrm{ng} / \mathrm{mL}$ (Online Supplementary Figure S3). First, we determined the SCADM1 levels in the peripheral blood of healthy volunteers, HTLV-1 carriers, and patients with HAM/TSP and various types of ATLL who had not been previously treated. The median values for plasma and serum sCADM1 from healthy volunteers were 181.3 $\mathrm{ng} / \mathrm{mL}$ and $173.5 \mathrm{ng} / \mathrm{mL}$ with $5^{\text {th }}-95^{\text {th }}$ percentile ranges of $142.7-234.0 \mathrm{ng} / \mathrm{mL}$ and $131.7-215.5 \mathrm{ng} / \mathrm{mL}$, respectively (Online Supplementary Figure S4A). There were neither a significant differences between the sCADM1 levels of males and females, nor amongst the age groups (Online Supplementary Figure S4B-C). In addition, the majority of HTLV-1 carriers and HAM/TSP patients had SCADM1 levels within the standard reference range (Figure 2). The median plasma sCADM1 concentration was slightly higher in smoldering-type ATLL patients $(210.2 \mathrm{ng} / \mathrm{mL})$ than in healthy volunteers $(181.3 \mathrm{ng} / \mathrm{mL}$ ), and it was elevated in chronic-type ATLL patients, with a median peak value of $267.1 \mathrm{ng} / \mathrm{mL}$ (Figure 2). The median plasma sCADM1 level was $1055.0 \mathrm{ng} / \mathrm{mL}$ (range: $173.6-6,931.0 \mathrm{ng} / \mathrm{mL}$ ) in acutetype ATLL patients, more than 5-fold higher than in the control group (Figure 2 and Online Supplementary Figure S5A). In addition, 2 of 10 patients with the unfavorable chronic-type ATLL35 had high levels of plasma sCADM1 (Online Supplementary Figure S6).

Next, we determined the association between plasma sCADM1 concentrations and other clinical and/or biochemical parameters in ATLL patients (Online Supplementary Figure S5A-E). Among these subjects, serum concentrations of sIL2R were higher in patients with all subtypes of ATLL compared to healthy volunteers, and concentrations increased with disease progression to acute-type or lymphoma-type ATLL (Online Supplementary Figure S5B). Importantly, while the patients with HAM/TSP showed significantly higher sIL2R levels compared with healthy volunteers, as reported previously, ${ }^{9}$ plasma sCADM1 levels were in the healthy range (Online Supplementary Figure S5A-B), confirming that SCADM1 is a specific marker for ATLL. Most patients with acute-type or lymphoma-type ATLL had elevated serum lactate dehydrogenase (LDH) levels (Online Supplementary Figure S5C). HTLV-1 proviral load (PVL) in the peripheral blood increased in smoldering-type ATLL patients and was elevated in chronic-type and acute-type ATLL, and was similar to white blood cell (WBC) concentrations (Online Supplementary Figure S5D-E). Serum sIL2R levels strongly correlated with plasma sCADM1 levels $(r=0.66, P<0.001)$ (Figure $3 \mathrm{~A}$ ), and moderate correlations were found between plasma sCADM1 and LDH levels ( $r=0.52$, $P<0.001$ ) (Figure $3 \mathrm{~B}$ ) as well as plasma sCADM1 and PVL levels in the peripheral blood $(\mathrm{r}=0.47, P<0.001)$ (Figure $3 \mathrm{C}$ ). The correlation between plasma sCADM1 and PVL levels was higher than that between plasma SCADM1 and the levels of oligoclonality of HTLV-1-infected clones (Online Supplementary Figure S7A-B). There was a weak correlation between WBC counts and plasma sCADM1 levels $(r=0.37$, $P<0.001$ ) (Figure 3D). Additionally, the increase in plasma concentrations of sCADM1 was much higher than the 
A
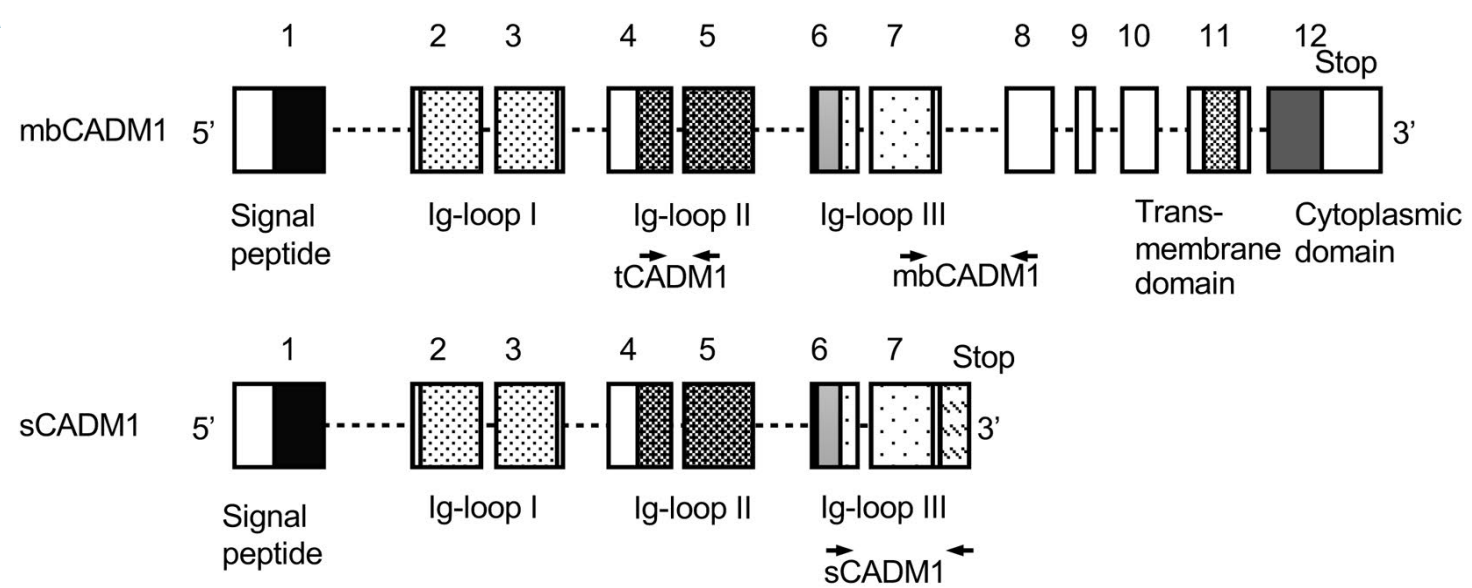

B
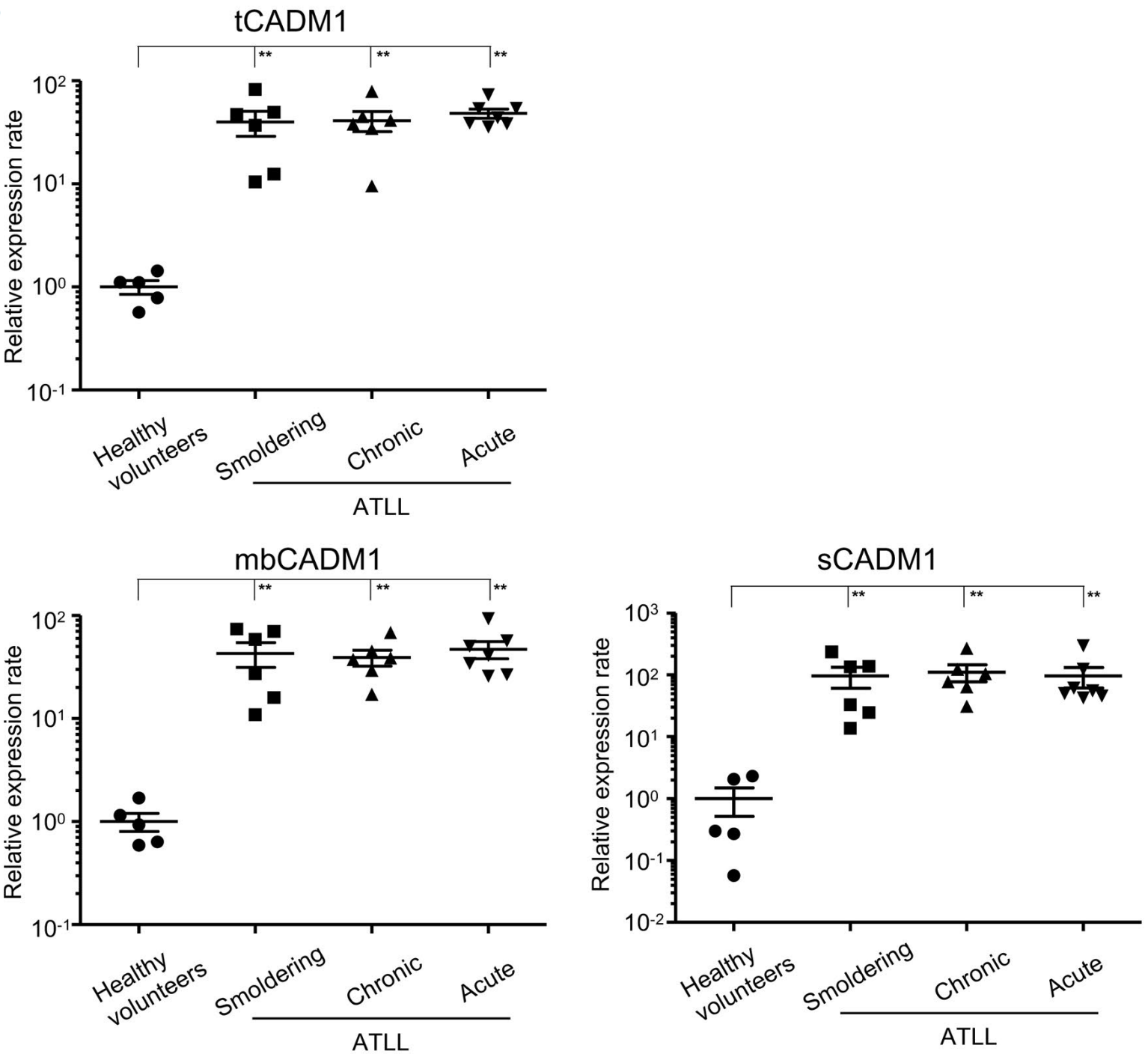

Figure 1. High expression of SCADM1 transcript in adult T-cell leukemia/lymphoma. (A) Schematic representation of the structure and domain organization of membrane-bound cell adhesion molecule 1 CADM1 (mbCADM1) and its soluble form (sCADM1). mbCADM1 contains an extracellular domain (exons 2-10) containing three immunoglobulin-like C2-type domains, a transmembrane domain (exon 11), and a cytoplasmic domain (exon 12). sCADM1 is generated by alternative splicing in which intron 7 is retained and an in-frame stop codon is found immediately downstream of the immunoglobulin-like domain. Arrows indicate the position and direction of primers used in RT-PCR reactions. The mbCADM1, sCADM1, and total CADM1 (tCADM1) transcripts were amplified using primers for exon 7 and exon 8 , exon 6 and intron 7, and exon 4 and exon 5, respectively. Numbers indicate exon numbers of the CADM1 gene. (B) Quantitative RT-PCR analysis of $t C A D M 1$, mbCADM1, and sCADM1 expression in CD4 ${ }^{+}$T lymphocytes from healthy volunteers $(n=5)$ and sorted CADM1 ${ }^{+}$cells from peripheral blood of patients with smoldering-type ( $n=6$ ), chronic-type $(n=6)$, and acute-type $(n=7)$ ATLL. The data were normalized to $\beta$-actin gene expression and expressed relative to a healthy control sample. Mean \pm standard deviation is shown, $* * P<0.01$ (Mann-Whitney $U$ test). 
increases in the percentages of $\mathrm{CD} 4^{+} \mathrm{CADM} 1^{+} \mathrm{T}$ cells, WBC concentrations, or serum sIL2R levels in acute-type ATLL patients compared to chronic-type ATLL patients (Online Supplementary Figure S8A-D). Notably, a strong positive correlation was found between the absolute number of circulating $\mathrm{CD}^{+} \mathrm{CADM} 1^{+} \mathrm{T}$ cells and sCADM1 concentration in the peripheral blood of chronic-type and acutetype ATLL patients (Online Supplementary Figure S9A-B). Given that sCADM1 expression did not significantly differ between chronic-type and acute-type ATLL (Figure 1B), the drastic increase in sCADM1 levels in acute-type ATLL is possibly ascribed to an increase in numbers of circulating tumor cells. Additionally, univariate and multivariate logistic regression analyses were performed to assess factors associated with aggressive ATLL compared with indolent ATLL. In the univariate analysis, the risk factors associated with aggressive type ATLL were sCADM1, sIL2R, LDH, and WBC (Table 1). On the other hand, in multivariate analysis, sCADM1 was the only risk factor associated with aggressive ATLL (Table 1). Furthermore, the Kaplan-Meier analysis in aggressive type ATLL patients showed a trend towards worse overall survival for patients with high sCADM1 (Online Supplementary Figure S10A-D, Online Supplementary Table S2). Taken together, these results suggest that sCADM1 has potential as a novel diagnostic biomarker in ATLL, and that it may be useful for monitoring disease progression.

The plasma sCADM1 level is a useful clinical indicator of the leukemic cell burden in ATLL patients

In order to investigate the clinical value of plasma sCADM1 concentration, we evaluated the levels of plasma sCADM1 along with sIL2R, LDH, PVL, and WBC in the peripheral blood of patients with various types of ATLL

Table 1. Univariate and multivariate analysis of factors predicting aggressiveness of adult T-cell leukemia/lymphoma.

\begin{tabular}{|c|c|c|c|c|c|c|}
\hline \multirow[b]{2}{*}{ Variable } & \multirow[b]{2}{*}{ OR } & \multicolumn{2}{|c|}{ Univariate analysis } & \multicolumn{3}{|c|}{ Multivariate analysis } \\
\hline & & $95 \%$ CI & $\boldsymbol{P}$ & OR & $95 \%$ CI & $\boldsymbol{P}$ \\
\hline sCADM1 & 5.46 & 3.13-9.54 & $<0.0001$ & 4.33 & $2.06-9.10$ & $<0.0001$ \\
\hline sIL2R $\alpha$ & 1.85 & $1.49-2.28$ & $<0.0001$ & 1.22 & 0.895-1.67 & 0.205 \\
\hline LDH & 6.93 & $3.48-13.8$ & $<0.0001$ & 2.22 & $0.944-5.21$ & 0.068 \\
\hline WBC & 1.72 & $1.22-2.44$ & 0.002 & 0.617 & $0.349-1.09$ & 0.096 \\
\hline $\mathrm{PVL}^{*}$ & 1.14 & $0.94-1.38$ & 0.158 & 0.855 & $0.611-1.2$ & 0.359 \\
\hline Age & 1.01 & $0.98-1.04$ & 0.385 & 1 & $0.957-1.05$ & 0.963 \\
\hline
\end{tabular}

For logistic regression analysis, numerical values of soluble for of cell adhesion molecule 1 (sCADM1), serum interleukin-2 receptor $\alpha$ (sIL2R $\alpha$ ), lactate dehydrogenase (LDH), white blood cell (WBC), and proviral load (PVL) were converted to base 2 logarithm transformation. The data from 49 aggressive type (40 acute-type and nine lymphoma-type) and 83 indolent type adult T-cell leukemia/lymphoma (ATLL) cases (18 chronic-type and 65 smoldering-type) (Online Supplementary Figure S5) were used in the analysis. *Fortytwo cases with missing values on PVL (eight acute-type, eight chronic-type, and 17 smoldering-type) and nine lymphoma type ATLL were excluded.

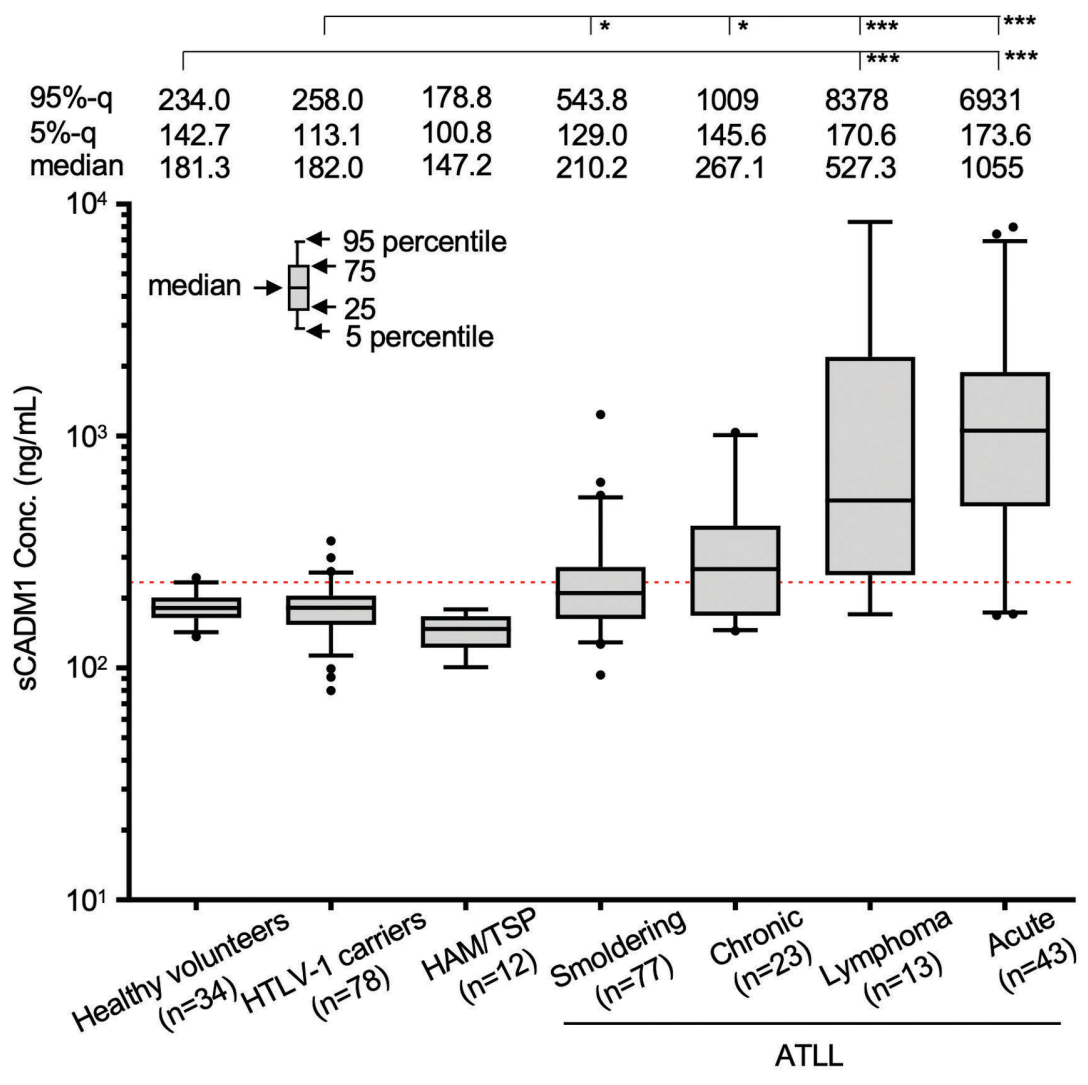

Figure 2. Plasma sCADM1 levels in adult T-cell leukemia/lymphoma patients examined by AlphaLISA. The plasma soluble form of cell adhesion molecule 1 (sCADM1) levels in 34 healthy volunteers, 78 human T-cell leukemia virus type 1 (HTLV-1) carriers, 12 HTLV-1-associated myelopathy/tropical spastic paraparesis (HAM/TSP) patients, 77 smoldering-type 23 chronic-type, 13 lymphoma-type, and 43 acute-type ATLL patients who were previously untreated were measured by AlphaLISA using anti-CADM1 antibodies. The box and whisker plots show the $5^{\text {th }}$, $25^{\text {th }}, 50^{\text {th }}$ (median), $75^{\text {th }}$, and $95^{\text {th }}$ percentile values, with outliers marked by solid dots. $* P<0.05, \quad * * P<0.01$ $* * * P<0.001$ versus healthy volunteers or HTLV-1 carriers (Kruskal-Wallis test/Dunn's multiple comparison test). Median and $5^{\text {th }}$ and $95^{\text {th }}$ percentile values are indicated at the top of each column. The dot line indicates the $95^{\text {th }}$ percentile of plasma SCADM1 in healthy subjects. 
before and after treatment with chemotherapy (Figure 4). Although the pre- and post-treatment samples were not derived from the same patients, median plasma sCADM1 levels were significantly decreased in the peripheral blood of patients with acute and lymphoma-type ATLL after chemotherapy compared to before treatment (Figure 4A). Additionally, the serum sIL2R levels in acute and lymphoma-type ATLL patients after chemothe-rapy were sig-

Table 2. Receiver operating characteristic (ROC) curve analysis of plasma SCADM1 concentrations.

\begin{tabular}{|c|c|c|c|c|c|c|}
\hline & Marker & Cut-off Point & Sensitivity & Specificity & AUC & $P$ \\
\hline ATLL (acute/lymphoma type) & sCADM1 (ng/mL) & 232.4 & 0.873 & 0.971 & 0.937 & $P<0.0001$ \\
\hline versus healthy & $\mathrm{WBC}(\times 100 / \mu \mathrm{L})$ & 92.5 & 0.630 & 0.969 & 0.786 & $P=0.0002$ \\
\hline volunteers & sIL2R $\alpha(\mathrm{U} / \mathrm{mL})$ & 494.5 & 1.0 & 1.0 & 1.0 & $P<0.0001$ \\
\hline ATLL (chronic type) & sCADMl (ng/mL) & 233.4 & 0.609 & 0.971 & 0.739 & $P=0.0024$ \\
\hline versus healthy & $\mathrm{WBC}(\times 100 / \mu \mathrm{L})$ & 76.5 & 1.0 & 0.875 & 0.981 & $P<0.0001$ \\
\hline volunteers & sIL2R $\alpha(\mathrm{U} / \mathrm{mL})$ & 470.0 & 1.0 & 1.0 & 1.0 & $P<0.0001$ \\
\hline ATLL (smoldering type) & sCADMl (ng/mL) & 214.6 & 0.481 & 0.912 & 0.657 & $P=0.0082$ \\
\hline versus healthy & $\mathrm{WBC}(\times 100 / \mu \mathrm{L})$ & 62.5 & 0.627 & 0.719 & 0.692 & $P=0.0020$ \\
\hline volunteers & sIL2R $\alpha(\mathrm{U} / \mathrm{mL})$ & 436.5 & 0.955 & 1.0 & 0.986 & $P<0.0001$ \\
\hline
\end{tabular}

The Youden index was used to determine cut-off values. ATLL: adult T-cell leukemia/lymphoma;AUC: area under the ROC curve; SCADM1: soluble form of cell adhesion molecule 1;WBC: white blood cell; sIL2R $\alpha$ : serum interleukin-2 receptor $\alpha$.
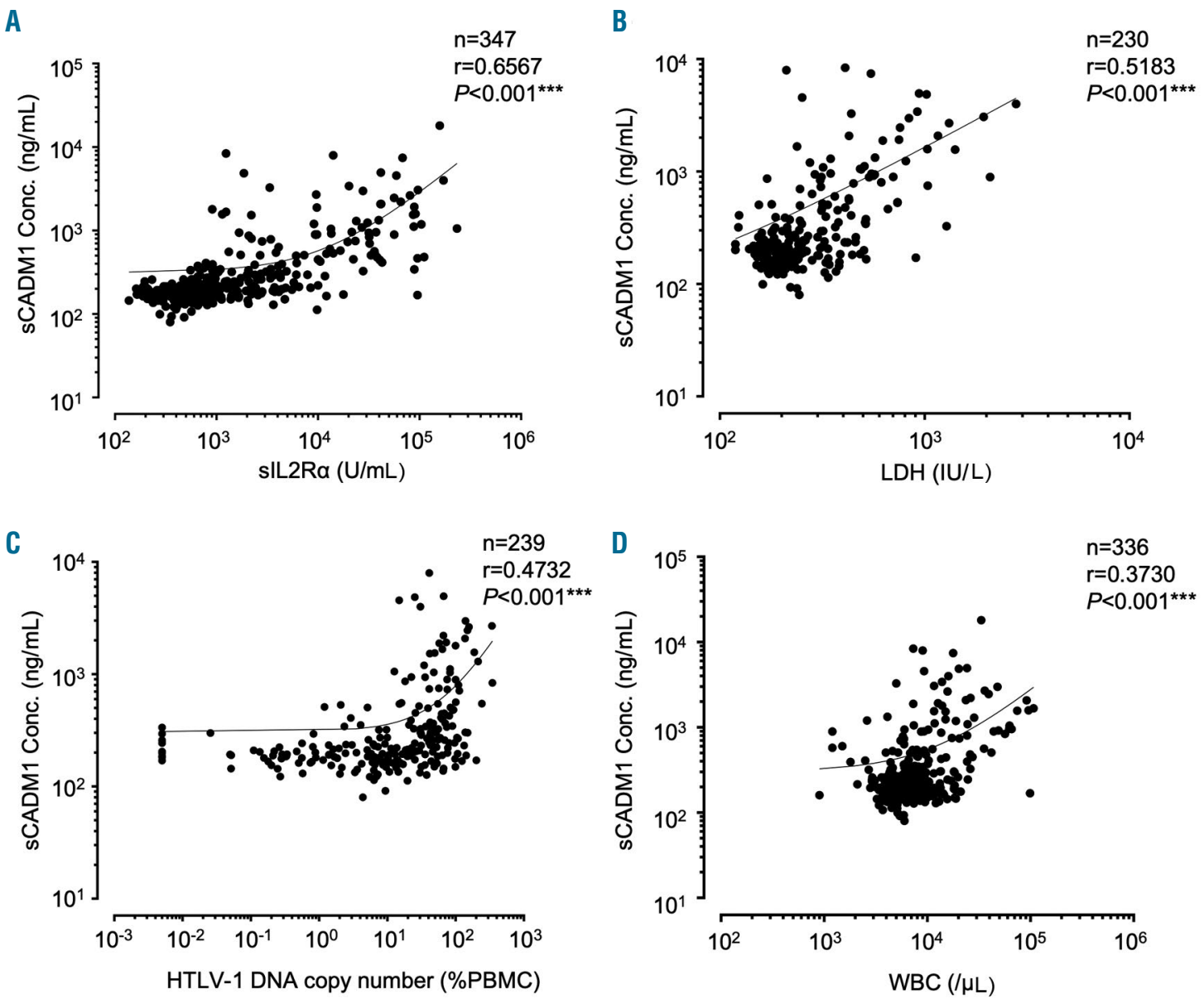

Figure 3. Association between plasma sCADM1 levels and various prognostic indicators in adult T-cell leukemia/lymphoma. (A) Scatter plot of plasma soluable form of cell adhesion molecule 1 (sCADM1) concentrations versus serum interleukin-2 receptor $\alpha$ (sIL2R $\alpha$ ) concentrations in 34 healthy volunteers, 78 human T-cell leukemia virus type 1 (HTLV-1) carriers, 77 smoldering-type, 55 chronic-type, 34 lymphoma-type, and 69 acute-type adult T-cell leukemia/lymphoma (ATLL) patients, including those who were previously untreated and treated with chemotherapy. Spearman correlation coefficient values ( $\mathrm{r}$ ) and $P$-values are shown on each of the graphs. (B) Scatter plot of plasma sCADM1 concentrations versus serum lactate dehydrogenase (LDH) concentrations in 45 HTLV-1 carriers, 69 smolderingtype, 38 chronic-type, 18 lymphoma-type, and 60 acute-type ATLL patients, including those who were previously untreated and treated with chemotherapy. Spearman correlation coefficient values $(r)$ and $P$-values are shown on each of the graphs. (C) Scatter plot of plasma sCADM1 concentrations versus proviral load (PVL) in 66 HTLV-1 carriers, 56 smoldering-type, 56 chronic-type, and 61 acute-type ATLL patients, including those who were previously untreated and treated with chemotherapy. Spearman correlation coefficient values $(r)$ and $P$-values are shown on each of the graphs. (D) Scatter plot of plasma sCADM1 concentrations versus white blood cell (WBC) counts in 32 healthy volunteers, 71 HTLV-1 carriers, 74 smoldering-type, 57 chronic-type, 33 lymphoma-type, and 69 acute-type ATLL patients, including those who were previously untreated and treated with chemotherapy. Spearman correlation coefficient values $(r)$ and $P$-values are shown on each of the graphs. 
A

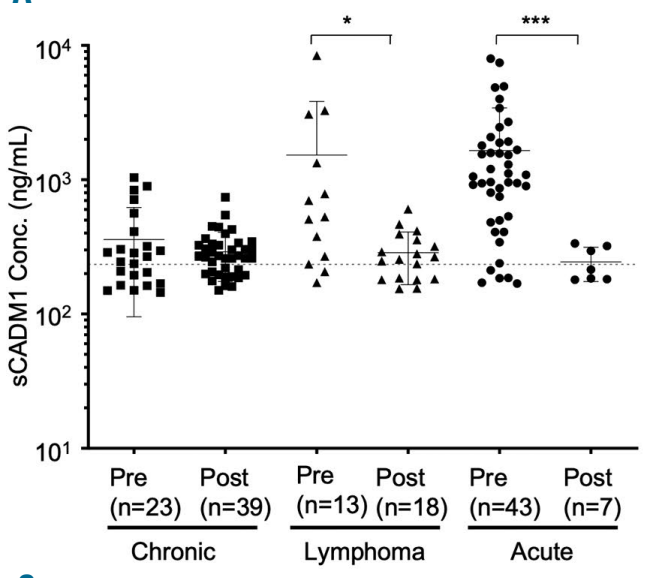

C

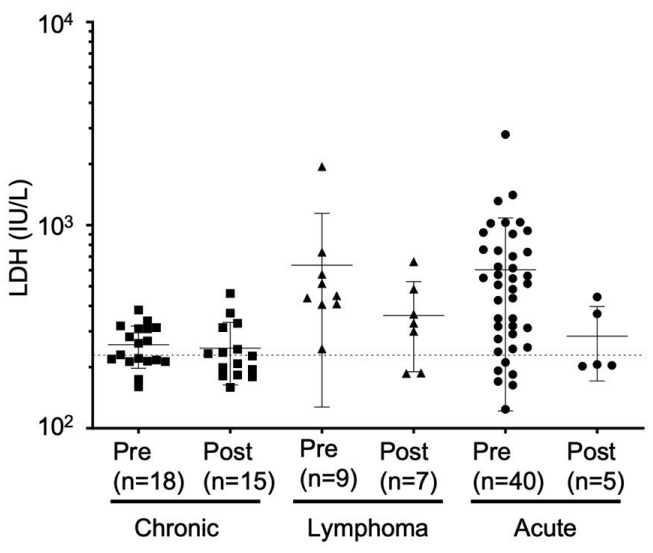

E
B

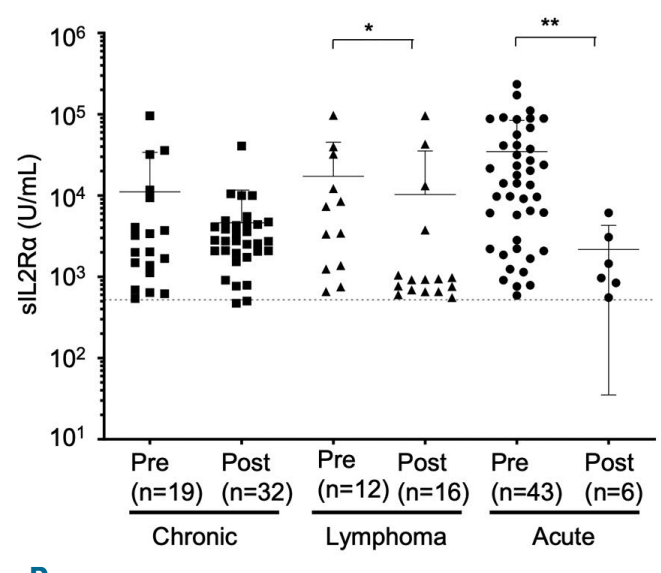

D

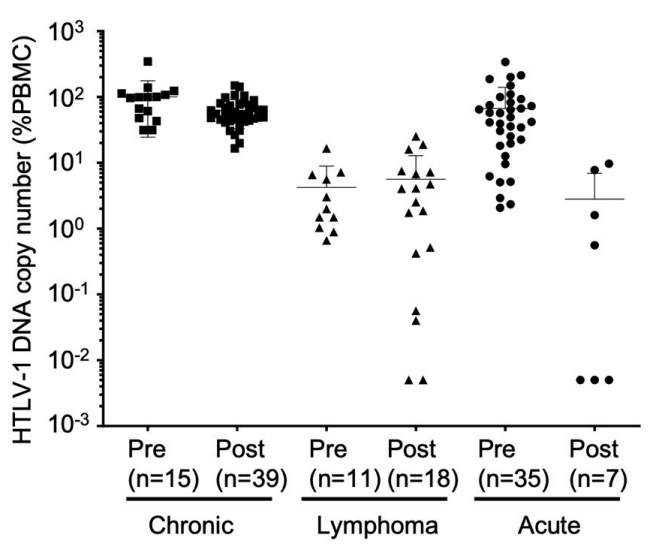

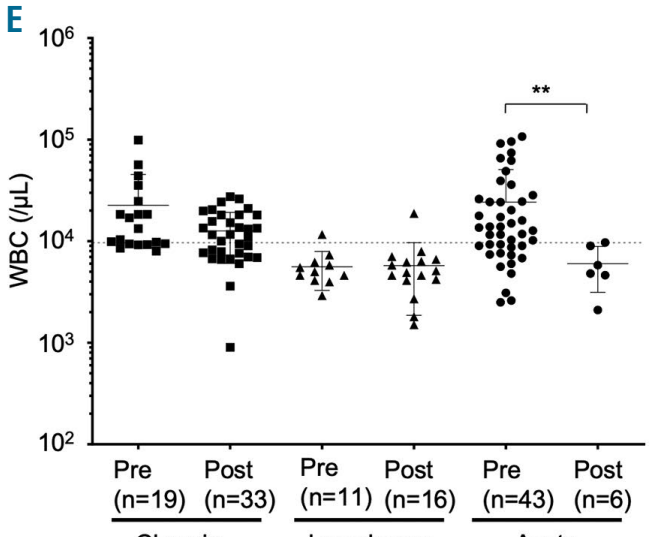

Chronic

Lymphoma

Acute

Figure 4. Plasma sCADM1 levels are related to treatment efficacy in adult T-cell leukemia/lymphoma patients. (A) Soluble form of cell adhesion molecule 1 (SCADM1) concentrations in the plasma of 23 chronic-type, 13 lymphoma-type, and 43 acute-type adult T-cell leukemia/lymphoma (ATLL) patients who were previously untreated and of 39 chronic-type, 18 lymphoma-type, and 7 acute-type ATLL patients who were previously treated with chemotherapy were measured by AlphaLISA. ${ }^{*} P<0.05, * * * P<0.001$ (Mann-Whitney $U$ test). The dot line indicates the $95^{\text {th }}$ percentile of plasma sCADM1 in healthy subjects. Note that the pre-treatment and post-treatment samples were derived from different individuals. (B) Serum interleukin-2 receptor $\alpha$ (sIL2R $\alpha$ ) concentrations in the serum of 19 chronictype, 12 lymphoma-type, and 43 acute-type ATLL patients who were previously untreated and of 32 chronic-type, 16 lymphoma-type, and six acute-type ATLL patients who were previously treated with chemotherapy. ${ }^{*} P<0.05, * * * P<0.001$ (Mann-Whitney U test). The dot line indicates the upper limit of normal serum sIL2R $\alpha$. The same samples as Figure 4A were used. Fifteen cases with missing values on sIL2R $\alpha$ (one lymphoma-type and four chronic-type in the pre-treatment group and one acute-type, two lymphoma-type, and seven chronic-type in the post-treatment group) were excluded. (C) Lactate dehydrogenase (LDH) concentrations in the serum of 18 chronic-type, nine lymphoma-type, and 40 acute-type ATLL patients who were previously untreated and of 15 chronic-type, seven lymphoma-type, and five acute-type ATLL patients who were previously treated with chemotherapy. ${ }^{*} P<0.05$ (Mann-Whitney $U$ test). The dot line indicates the upper limit of normal serum LDH. The same samples as Figure 4A were used. Forty-nine cases with missing values on LDH (three acute-type, four lymphoma-type, and five chronic-type in the pre-treatment group and two acute-type, 11 lymphoma-type, and 24 chronic-type in the post-treatment group) were excluded. (D) Human T-cell leukemia virus type 1 (HTLV-1) proviral load (PVL) in 15 chronic-type, 11 lymphoma-type, and 35 acute-type ATLL patients who were previously untreated and in 39 chronic-type, 18 lymphoma-type, and 7 acute-type ATLL patients who were previously treated with chemotherapy. $* * P<0.01$ (Mann-Whitney U test). The same samples as Figure $4 \mathrm{~A}$ were used. Eighteen cases with missing values on PVL (eight acute-type, two lymphoma-type, and eight chronic-type in the pre-treatment group) were excluded. (E) White blood cell counts (WBC) counts in 19 chronic-type, 11 lymphoma-type, and 43 acute-type ATLL patients who were previously untreated and in 33 chronic-type, 16 lymphoma-type, and 6 acute-type ATLL patients who were previously treated with chemotherapy. $* * * P<0.001$ (Mann-Whitney $U$ test). The dot line indicates the upper limit of normal for WBC counts. The same samples as Figure 4A were used. Thirteen cases with missing values on WBC counts (two lymphoma-type and four chronic-type in the pre-treatment group and one acute-type, two lymphoma-type, and four chronic-type in the post-treatment group) were excluded. 
nificantly lower than before treatment (Figure 4B). A significant reduction in serum LDH, PVL, and WBC after chemotherapy was also observed in acute-type ATLL patients (Figure 4C-E). Plasma sCADM1 levels were examined in three patients with acute-type ATLL who received
allo-HSCT (Online Supplementary Table S3). Although all three patients exhibited elevated levels of serum sIL2R $\alpha, 2$ of the 3 patients showed normal (case 3 ) or slightly high levels (case 1) of plasma sCADM1. Notably, in one patient (case 2) with elevated levels of both sIL2R and sCADM1,
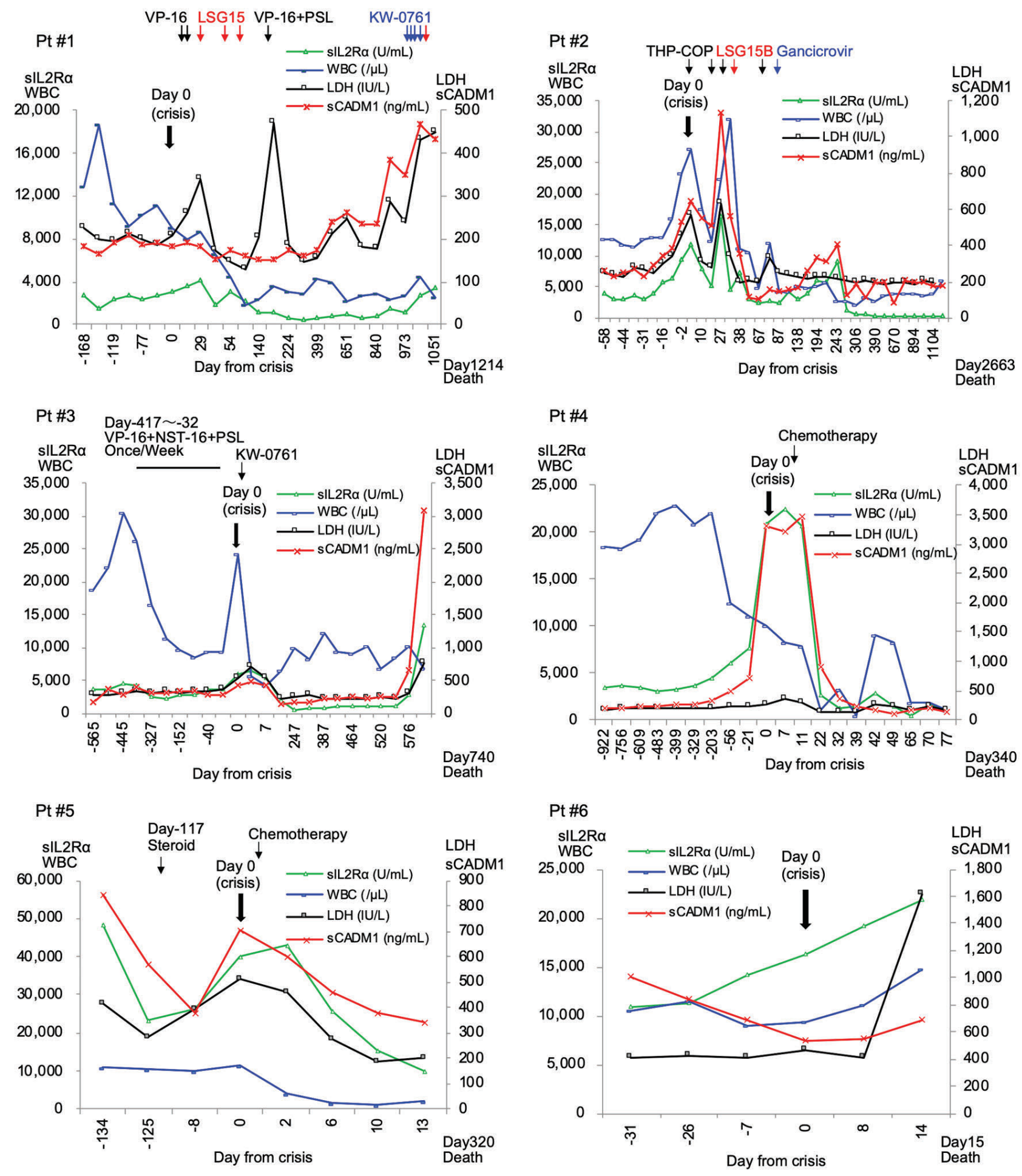

Figure 5. Plasma sCADM1 is useful for monitoring disease progression in adult T-cell leukemia/lymphoma patients. Plasma soluble form of cell adhesion molecule 1 (sCADM1) concentrations along with various prognostic parameters including serum interleukin-2 receptor $\alpha$ (sIL2R $\alpha$ ), lactate dehydrogenase (LDH), and white blood count (WBC) count were monitored in six patients with adult T-cell leukemia/lymphoma (ATLL) before and after acute crisis (day 0). The drug regimen is indicated at the top of the figure: VP-16, etoposide; LSG15, VCAP-AMP-VECP (vincristine, cyclophosphamide, doxorubicin, and prednisone [VCAP], doxorubicin, ranimustine, and prednisone [AMP], and vindesine, etoposide, carboplatin, and prednisone [VECP]); PSL: prednisolone; KW-0761: mogamulizumab; THP-COP: pirarubicin, cyclophosphamide, vincristine, and prednisolone. 
recurrence of ATLL occurred shortly after allo-HSCT. These results suggest that mea-surement of plasma sCADM1 levels may be useful in monitoring response to chemotherapy as well as in predicting recurrence of ATLL after allo-HSCT.

In order to further evaluate the relationship between plasma sCADM1 concentration and treatment response or the leukemic cell burden during the clinical course of ATLL patients, six ATLL patients were followed over a period of 45 to 1,219 days during treatment to assess changes in sCADM1 levels along with WBC, LDH, and sIL2R in the peripheral blood (Online Supplementary Table S4, Figure 5). In all of the patients except one (Pt \#6), the levels of sCADM1 and sIL2R showed similar changes during the observation period, and importantly, increases in sCADM1 appears to correlate with poor outcome (Pt \#1 and \#3). While changes in LDH levels were related to changes in levels of sCADM1 in four patients (Pt\#1, \#2, \#3, and \#5), WBC counts did not appear to correlate with the levels of sCADM1 and sIl2R (Figure 5). Moreover, the sCADM1 level was also evaluated during the asymptomatic period and ATLL disease progression in each patient. In most cases, changes in the levels of sCADM1 from the asymptomatic state to ATLL stages showed a similar tendency to those observed in PVL (Online Supplementary Figure S11-12). In one case (MK1006), we observed a marked increase of sCADM1 from the smoldering to the lymphoma-type, which coincided with an increase in sIL2R (from 2,080 $\mathrm{U} / \mathrm{mL}$ to $36,300 \mathrm{U} / \mathrm{mL}$ ), whereas PVL was not increased in the peripheral blood (Online Supplementary Figure S12).

As a few HTLV-1 carriers had an elevated plasma sCADM1 concentration compared to healthy subjects (Figure 2), we assessed whether sCADM1 levels are a predictive marker for the development of ATLL. Because $\mathrm{PVL}>4 \%$ is a known predictor for the development of ATLL, ${ }^{36}$ we compared the sCADM1 concentrations of asymptomatic HTLV-1 carriers with PVL $>4 \%$ and PVL $<4 \%$, along with those who later progressed to ATLL (Online Supplementary Figure S13). There were no differences in plasma SCADM1 levels between the high and low PVL groups and no association was found between sCADM1 level and PVL in the univariate analysis (Online Supplementary Figure S13, Online Supplementary Table S5), although a slightly higher median SCADM1 concentration was observed in HTLV-1 carriers who later developed ATLL compared to the other groups (Online Supplementary Figure S13). Thus, these data suggest that while measurement of plasma sCADM1 alone may not be used to predict the progression of HTLV-1 carriers to indolent ATLL, it may be helpful for monitoring disease progression to an aggressive state, leukemic cell burden, and/or treatment response in ATLL patients. Finally, in order to assess the diagnostic efficiency of the measurement of plasma sCADM1, we examined receiver operating characteristics (ROC) in patients with different subtypes of ATLL and in healthy controls. The area under the ROC curve of SCADM1 was 0.94 for acute and lymphoma-type, 0.74 for chronic-type, and 0.66 for smoldering-type ATLL (Table 2), suggesting that sCADM1 is a promising functional biomarker for the diagnosis of aggressive ATLL.

\section{Discussion}

In this study, we used AlphaLISA technology to measure sCADM1 concentrations in the peripheral blood.
Although ELISA is a well-known technique to measure the plasma concentration of target proteins, we found that detection sensitivity of plasma sCADM1 in our AlphaLISA system was enhanced $\sim 10$-fold compared to a conventional ELISA method (data not shown). By using this system, we successfully determined the range of plasma sCADM1 concentrations in healthy subjects and cut-off points for plasma sCADM1 between healthy and ATLL patients.

The disease progression in ATLL patients may involve the accumulation of genetic alterations and clonal expansion of ATLL cells. Although recent studies have identified somatic mutations that act as drivers during ATLL progression, ${ }^{37,38}$ the application of this genetic information towards diagnosing patients is not easy, because the genomic abnormalities of ATLL cells are very complicated. In addition, clonality assessment is currently high-cost and time-consuming. Therefore, the development of effective methods for predicting disease progression is urgently needed.

CADM1 is transcriptionally upregulated in HTLV-1infected $\mathrm{T}$ cells and ATLL cells in almost all cases, ${ }^{19,21,39,40}$ and in this study we found that the same CADM1 promoter appears to activate the expression of both sCADM1 and the membrane-bound form of CADM1 transcripts in ATLL. On the other hand, plasma sCADM1 levels were drastically increased in acute stage ATLL compared to chronic stage ATLL (median 1,066.7 ng/mL vs. 204.0 $\mathrm{ng} / \mathrm{mL}$, respectively), whereas the median percentages of $\mathrm{CD}^{+}{ }^{+} \mathrm{CADM} 1^{+} \mathrm{T}$ cells were $78.8 \%$ and $43.1 \%$ in acute and chronic-type ATLL, respectively (Online Supplementary Figure S8). Moreover, we observed increases in plasma sCADM1 levels during follow-ups with patients, which were accompanied by poor patient outcomes, whereas WBC counts were not changed (Figure 5). As multivariate analysis of several blood biomarkers for ATLL identified sCADM1 as the only independent serum marker for aggressive ATLL with significant differences (Table 1), plasma sCADM1 may be a potential risk factor of the aggressiveness of ATLL cells. In addition, while sIL2R $\alpha$ is secreted not only by leukemia cells but also during various inflammatory responses, ${ }^{15,41}$ plasma sCADM1 was not increased in HAM/TSP patients or in a patient with GvHD after allo-HSCT for ATLL. Therefore, combined sCADM1 and sIL2R $\alpha$ measurements may become a promising method for more accurately diagnosing ATLL development in HAM/TSP patients, or discriminating ATLL relapse and GvHD following allo-HSCT for ATLL. In the current clinical setting, assessment of treatment efficacy in ATLL is based on serum biochemical parameters such as sIL2R $\alpha, \mathrm{LDH}$, and calcium levels, and clinical findings including computed tomography and positron emission tomography examination results, however, these tests are unable to determine the depth of response to treatment, which can be evaluated by detection of MRD. Given that sCADM1 seems to be a specific biomarker for ATLL, the measurement of plasma sCADM1 may be a useful in measuring the depth of response to therapy, and a change in the sCADM1 plasma level may become an important cli-nical criteria for not only determining the transplant adap-tability, but also determining the choice of consolidation or maintenance treatments and their periods.

It has been reported that SCADM1 can bind to the extracellular domain of CADM1 through an interaction between their Ig domains. ${ }^{27} \mathrm{CADM} 1$ contains a PSD95/Dlg/ZO-1 (PDZ) domain that interacts with $\mathrm{T}$ lymphoma invasion 
and metastasis 1 (TIAM1), and induces signaling to actin filaments, leading to cell structure remodeling. ${ }^{42}$ sCADM1 may activate this TIAM1-actin signaling to modulate cellular functions. It has also been reported that CADM1 expression on tumor cells enhances the cytotoxic activities of cytotoxic T cells or natural killer cells via an interaction with Class I MHC-restricted T-cell-associated molecule (CRTAM) ${ }^{40,43}$ The CRTAM-CADM1 interaction acts as a costimulator of T-cell receptor (TCR) signaling that may help to eliminate tumor cells. Because sCADM1 has been reported to bind to the extracellular domain of CADM1 on the cell surface and inhibit CADM1 homophilic interactions ${ }^{27}$ it is possible that sCADM1 may modulate immune responses under certain conditions. Future studies of the biological role of sCADM1 in anti-tumor immune responses will be important to obtain a better understanding of the molecular mechanisms of the immunological abnormalities in ATLL. ${ }^{44}$

To date, there has been no report identifying sCADM1 in cancers; however, it has been reported that CADM1 shedding was elevated in the lung epithelial cells of patients with idiopathic interstitial pneumonias ${ }^{45}$ or pulmonary emphysema, ${ }^{46}$ as well as in the pancreatic islets of patients with type 2 diabetes mellitus, ${ }^{47}$ which was accompanied by a decrease in full-length CADM1 levels and may be associated with the disruption of cell polarity and cell apoptosis. ${ }^{45,46}$ In ATLL, plasma sCADM1 levels drastically increased from chronic to acute-type ATLL with no apparent reduction in the expression of full-length CADM1, suggesting that sCADM1 may play a role in the malignant progression of ATLL.

In summary, sCADM1 is a promising biomarker not only for monitoring the leukemic burden of ATLL patients, but also for predicting disease status. Thus, sCADM1 measurement may be valuable for the diagnosis of ATLL.

\section{Disclosures}

No conflicts of interests to disclose.

\section{Contributions}

SN performed research, analyzed data and wrote the manuscript; CS, AN, KSa, MY, IN, and MS performed research and analyzed data; TK, KSh, YK, TH, AK, ST, NN, MI, YS, $K M o, M A, K M a, E S, A O, K S h$, and $T W$ provided clinical information and samples. YU, TM, and AI provided critical reagents; $A U$ provided clinical information and samples, and critical evaluation of the manuscript; and KM conceived and designed the study, directed and supervised the research, and wrote the manuscript.

\section{Acknowledgments}

The authors are grateful to Dr. Hidekatsu Iha (Oita University, Japan), Dr. Yasuaki Yamada (Nagasaki University, Japan), and Dr. Naomichi Arima (Kagoshima University, Japan) for providing cell lines.

\section{Funding}

This work was supported in part by Grant-in-Aid for Scientific Research (B) (25293081 and 17H03581) (KM) from the Japanese Society for the Promotion of Science (JSPS), by the Platform of Supporting Cohort Study and Biospecimen Analysis, Grant-in-Aid for Scientific Research on Innovative Areas (16H06277) (TW), and by the Takeda Science Foundation (KM).

\section{References}

1. Uchiyama T, Yodoi J, Sagawa K, Takatsuki $\mathrm{K}$, Uchino H. Adult T-cell leukemia: clinical and hematologic features of 16 cases. Blood. 1977:50(3):481-492

2. Poiesz BJ, Ruscetti FW, Gazdar AF, Bunn PA, Minna JD, Gallo RC. Detection and isolation of type $C$ retrovirus particles from fresh and cultured lymphocytes of a patient with cutaneous T-cell lymphoma. Proc Natl Acad Sci USA. 1980;77(12):7415-7419.

3. Bangham CRM, Matsuoka M. Human T-cell leukaemia virus type 1: parasitism and pathogenesis. Philos Trans R Soc Lond B Biol Sci. 2017;372:1732

4. Watanabe T. Current status of HTLV-1 infection. Int J Hematol. 2011;94(5):430-434.

5. Utsunomiya A, Choi I, Chihara D, Seto M. Recent advances in the treatment of adult Tcell leukemia-lymphomas. Cancer Sci. 2015;106(4):344-351.

6. Mehta-Shah N, Ratner L, Horwitz SM. Adult T-cell leukemia/lymphoma. J Oncol Pract. 2017;13(8):487-492.

7. Phillips AA, Harewood JCK. Adult T cell leukemia-lymphoma (ATL): state of the art. Curr Hematol Malig Rep. 2018;13(4):300307.

8. Kamihira S, Atogami S, Sohda H, Momita S, Yamada Y, Tomonaga M. Significance of soluble interleukin-2 receptor levels for evaluation of the progression of adult T-cell leukemia. Cancer. 1994;73(11):2753-2758.

9. Toledo-Cornell C, Santos S, Orge G, Glesby MJ, Carvalho EM. Soluble IL-2 receptor and beta- 2 microglobulin as possible serologic markers of neurologic disease in HTLV-1 infection. J Med Virol. 2014;86(2):315-321.

10. Nakamura Y, Tanaka Y, Tanaka M, et al. Soluble interleukin-2 receptor index predicts the development of acute graft-versus-host disease after allogeneic hematopoietic stem cell transplantation from unrelated donors. Int J Hematol. 2016;103(4):436-443

11. Budde H, Papert S, Maas JH, et al. Prediction of graft-versus-host disease: a biomarker panel based on lymphocytes and cytokines. Ann Hematol. 2017;96(7):1127-1133.

12. Fuji $S$, Inoue $Y$, Utsunomiya $A$, et al. Pretransplantation anti-CCR4 antibody Mogamulizumab against adult T-cell leukemia/lymphoma is associated with significantly increased risks of severe and corticosteroid-refractory graft-versus-host disease, nonrelapse mortality, and overall mortality. J Clin Oncol. 2016;34(28):3426-3433.

13. Kawano N, Kuriyama T, Yoshida S, et al. The Impact of a humanized CCR4 antibody (Mogamulizumab) on patients with aggressive-type adult T-cell leukemia-lymphoma treated with allogeneic hematopoietic stem cell transplantation. J Clin Exp Hematop. 2017;56(3):135-144

14. Cook LB, Fuji S, Hermine O, et al. Revised adult T-cell leukemia-lymphoma International Consensus Meeting Report. J Clin Oncol. 2019:37(8):677-687.

15. Murakami S. Soluble interleukin-2 receptor in cancer. Front Biosci. 2004;9:3085-3090.

16. Bien E, Balcerska A. Serum soluble interleukin 2 receptor alpha in human cancer of adults and children: a review. Biomarkers.
2008;13(1):1-26

17. Kuramochi $M$ Fukuhara $H$, Nobukuni $T$, et al. TSLC1 is a tumor-suppressor gene in human non-small-cell lung cancer. Nat Genet. 2001:27(4):427-430.

18. Pletcher MT, Nobukuni T, Fukuhara $\mathrm{H}$, et al. Identification of tumor suppressor candidate genes by physical and sequence mapping of the TSLC1 region of human chromosome 11q23. Gene. 2001;273(2):181-189.

19. Sasaki H, Nishikata I, Shiraga $T$, et al. Overexpression of a cell adhesion molecule, TSLC1, as a possible molecular marker for acute-type adult T-cell leukemia. Blood. 2005;105(3):1204-1213.

20. Dewan MZ, Takamatsu N, Hidaka T, et al. Critical role for TSLC1 expression in the growth and organ infiltration of adult T-cell leukemia cells in vivo. J Virol. 2008, 82(23):11958-11963.

21. Nakahata S, Saito Y, Marutsuka K, et al. Clinical significance of CADM1/TSLC1/IgSF4 expression in adult T-cell leukemia/lymphoma. Leukemia. 2012;26(6):1238-1246.

22. Kobayashi S, Watanabe E, Ishigaki T, et al. Advanced human T-cell leukemia virus type 1 carriers and early-stage indolent adult Tcell leukemia-lymphoma are indistinguishable based on CADM1 positivity in flow cytometry. Cancer Sci. 2015;106(5):598-603.

23. Kobayashi S, Nakano K, Watanabe E, et al. CADM1 expression and stepwise downregulation of CD7 are closely associated with clonal expansion of HTLV-I-infected cells in adult T-cell leukemia/lymphoma. Clin Cancer Res. 2014;20(11):2851-2861. 
24. Paulsson K, An Q, Moorman AV, et al. Methylation of tumour suppressor gene promoters in the presence and absence of transcriptional silencing in high hyperdiploid acute lymphoblastic leukaemia. $\mathrm{Br}$ JHaematol. 2009;144(6):838-847.

25. Murray PG, Fan Y, Davies G, et al. Epigenetic silencing of a proapoptotic cell adhesion molecule, the immunoglobulin superfamily member IGSF4, by promoter $\mathrm{CpG}$ methylation protects Hodgkin lymphoma cells from apoptosis. Am J Pathol. 2010;177(3):1480-1490.

26. Fu L, Gao Z, Zhang X, et al. Frequent concomitant epigenetic silencing of the stressresponsive tumor suppressor gene CADM1, and its interacting partner DAL-1 in nasal NK/T-cell lymphoma. Int J Cancer. 2009; 124(7):1572-1578.

27. Koma Y, Ito A, Wakayama T, et al. Cloning of a soluble isoform of the SgIGSF adhesion molecule that binds the extracellular domain of the membrane-bound isoform. Oncogene. 2004;23(33):5687-5692.

28. Hagiyama $M$, Ichiyanagi $N$, Kimura $K B$, Murakami Y, Ito A. Expression of a soluble isoform of cell adhesion molecule 1 in the brain and its involvement in directional neurite outgrowth. Am J Pathol. 2009; 174(6):2278-2289.

29. Nagara Y, Hagiyama M, Hatano N, et al. Tumor suppressor cell adhesion molecule 1 (CADM1) is cleaved by adisintegrin and metalloprotease 10 (ADAM10) and subsequently cleaved by $\mathrm{\gamma}$-secretase complex. Biochem Biophys Res Commun. 2012; 417(1):462-467.

30. Moiseeva EP, Leyland ML, Bradding P. CADM1 is expressed as multiple alternatively spliced functional and dysfunctional isoforms in human mast cells. Mol Immunol. 2013;53(4):345-354.

31. Shirakabe K, Omura T, Shibagaki Y, et al. Mechanistic insights into ectodomain shed- ding: susceptibility of CADM1 adhesion molecule is determined by alternative splicing and O-glycosylation. Sci Rep. 2017; 7:46174.

32. Eglen RM, Reisine T, Roby P, et al. The use of AlphaScreen technology in HTS: current status. Curr Chem Genomics. 2008;1:2-10.

33. McGiven JA, Sawyer J, Perrett LL, et al. A new homogeneous assay for high throughput serological diagnosis of brucellosis in ruminants. J Immunol Methods. 2008; 337(1):7-15.

34. Kurosawa G, Akahori Y, Morita M, et al Comprehensive screening for antigens overexpressed on carcinomas via isolation of human $\mathrm{mAbs}$ that may be therapeutic. Proc Natl Acad Sci U S A. 2008;105(20):7287 7292.

35. Tsukasaki K, Tobinai K. HTLV-1-associated T-cell diseases. In: Francine F, editor. T-cell lymphomas. New York: Humana; 2013. pp. 113-135.

36. Iwanaga M, Watanabe $T$, Utsunomiya A, et al. Human T-cell leukemia virus type I (HTLV-1) proviral load and disease progression in asymptomatic HTLV-1 carriers: a nationwide prospective study in Japan. Blood. 2010;116(8):1211-1219.

37. Kataoka K, Nagata Y, Kitanaka A, et al Integrated molecular analysis of adult $\mathrm{T}$ cell leukemia/lymphoma. Nat Genet. 2015; 47(11):1304-1315

38. Shah UA, Chung EY, Giricz O, et al. North American ATLL has a distinct mutationa and transcriptional profile and responds to epigenetic therapies. Blood. 2018; 132(14):1507-1518.

39. Sarkar B, Nishikata I, Nakahata S, et al. Degradation of 477 by autophagy contributes to CADM1 overexpression in ATLL cells through the activation of NF-KB. Sci Rep. 2019;9(1):3491.

40. Manivannan K, Rowan AG, Tanaka Y, Taylor GP, Bangham CR. CADM1/TSLC1 identifies HTLV-1-infected cells and determines their susceptibility to CTL-mediated lysis. PLoS Pathog. 2016;12(4):e1005560.

41. Seidler S, Zimmermann HW, Weiskirchen R, Trautwein C, Tacke F. Elevated circulating soluble interleukin-2 receptor in patients with chronic liver diseases is associated with non-classical monocytes. BMC Gastroenterol. 2012;12:38.

42. Masuda M, Maruyama T, Ohta $T$, et al. CADM1 interacts with Tiam1 and promotes invasive phenotype of human T-cell leukemia virus type I-transformed cells and adult T-cell leukemia cells. J Biol Chem 2010;285(20):15511-15522.

143. Boles KS, Barchet W, Diacovo T, Cella M Colonna $M$. The tumor suppressor TSLC1/NECL-2 triggers NK-cell and CD8+ T-cell responses through the cell-surface receptor CRTAM. Blood. 2005;106(3):779. 786.

44s. Kannagi M, Hasegawa A, Nagano Y Kimpara S, Suehiro Y. Impact of host immunity on HTLV-1 pathogenesis: potential of Tax-targeted immunotherapy against ATL. Retrovirology. 2019;16(1):23.

45. Yoneshige A, Hagiyama M, Inoue $T$, et al Increased ectodomain shedding of cell adhesion molecule 1 as a cause of type II alveolar epithelial cell apoptosis in patients with idiopathic interstitial pneumonia. Respir Res. 2015;16:90.

46. Mimae T, Hagiyama M, Inoue $T$, et al Increased ectodomain shedding of lung epithelial cell adhesion molecule 1 as a cause of increased alveolar cell apoptosis in emphysema. Thorax. 2014;69(3):223231.

47. Inoue T, Hagiyama M, Yoneshige A, et al. Increased ectodomain shedding of cell adhesion molecule 1 from pancreatic islets in type 2 diabetic pancreata: correlation with hemoglobin A1c levels. PLoS One. 2014; 9(6):e100988. 